López, L.; Oriol, M. Q-Rapids: Quality-Aware Rapid Software Development: an H2020 Project. A: International Conference on Product-Focused Software Process Improvement. "Product-Focused Software Process Improvement, 20th International Conference, PROFES 2019: Barcelona, Spain, November 27-29, 2019: proceedings". Berlín: Springer, 2019, p. 608-612.

The final authenticated version is available online at https://doi.org/10.1007/978-3-030-35333-9_46

\title{
Q-Rapids: Quality-Aware Rapid Software Development - An H2020 Project
}

\author{
Lidia López ${ }^{[0000-0002-6901-9223]}$, Marc Oriol ${ }^{[0000-0003-1928-7024]}$ \\ Universitat Politècnica de Catalunya (UPC), Barcelona, Spain \\ \{llopez, moriol\}@essi.upc.edu
}

\begin{abstract}
This work reports the objectives, current state, and outcomes of the QRapids H2020 project. Q-Rapids (Quality-Aware Rapid Software Development) proposes a data-driven approach to the production of software following very short development cycles. The focus of Q-Rapids is on quality aspects, represented through quality requirements. The Q-Rapids platform, which is the tangible software asset emerging from the project, mines software repositories and usage $\operatorname{logs}$ to identify candidate quality requirements that may ameliorate the values of strategic indicators like product quality, time to market or team productivity. Four companies are providing use cases to evaluate the platform and associated processes.
\end{abstract}

Keywords: software quality, data-driven requirements engineering, rapid software development, quality requirements.

\section{Introduction}

The Q-Rapids project (Quality-Aware Rapid Software Development) is a 3-year project funded by the European Union's Horizon 2020 research and innovation programme under grant agreement No 732253. It started in November 2016 and finishes in October 2019. The project website is at https://www.q-rapids.eu/.

The Q-Rapids consortium is composed of serven partners from five European countries, namely three research organisations, one SME, two mid-caps and one corporative:

- Universitat Politècnica de Catalunya, Spain, acting as coordinator.

- University of Oulu, Finland.

- IESE Fraunhofer, Germany.

- Bittium Wireless OY, Finland.

- Softeam, France.

- ITTI SP ZOO, Poland.

- Nokia Solutions and Networks OY, Finland.

As a result, the consortium combines long research tradition in software development and cutting-edge technological knowhow in versatile ICT sectors. 


\section{Project Goals}

Fig. 1 summarizes the concept of the Q-Rapids project. It shows the full data-driven cycle. Quality requirements (QRs) are incrementally elicited, refined and improved based on data gathered from software repositories, project management tools, system usage and quality of service. This data is analysed and aggregated into quality-related key strategic indicators (e.g., time-to-market delay related to not including the implementation of a given $\mathrm{QR}$ in the next development cycle) which are presented to decision makers using a highly informative dashboard. QRs scheduled for the next cycle are integrated with functional requirements for their uniform treatment in the rapid software development life cycle. See [1] for more details.

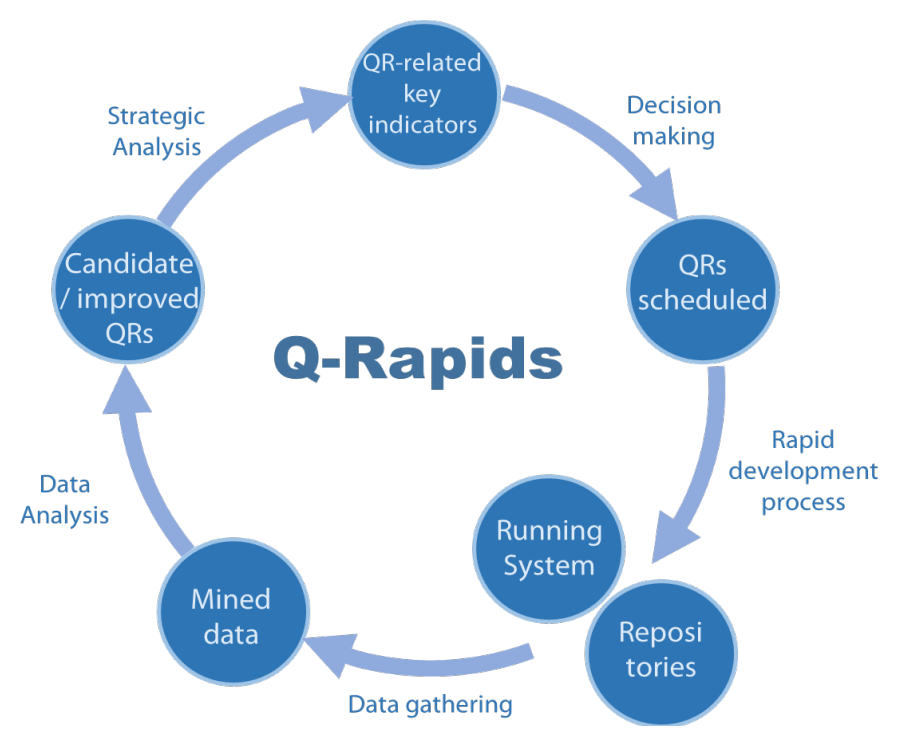

Fig. 1. Q-Rapids concept

The general objectives of the project are:

- Objective GO1. Improve the quality levels of software products and services with the support of data-driven IT infrastructure and associated methods and techniques.

- Objective GO2. Increase the productivity of the software life cycle with a seamless integration of quality requirements into the development process.

- Objective GO3. Reduce the time to market of software products and services by making optimal decisions based on strong evidence and solid experience-based decision-making models.

These general objectives are made actionable through several scientific objectives:

- Objective SO1. To provide methods to systematically collect and analyse runtime and development time data to improve software quality.

- Objective SO2. To define a rapid software life cycle process that integrates quality requirements and functional requirements into a holistic method. 
- Objective SO3. To provide quality-related key strategic indicators to support decision makers in managing the development process from a quality-aware perspective.

- Objective SO4. To implement adequate tool support to a quality-aware software life cycle.

The outcome of all these objectives will be a validated Q-Rapids framework: a quality-aware rapid software development process supported by advanced tools and methods.

\section{$3 \quad$ Project Use Cases}

As usual in $\mathrm{H} 2020$ research and innovation actions, the feasibility of Q-Rapids is being demonstrated using a significant portfolio of diverse use cases to demonstrate its potential. From a methodological perspective, the use cases play a two-fold role in this project: 1) They help to collect empirical data needed to solidify the objectives of the project and to create the baseline upon which the methods and tools are defined, and 2) they enable the assessment of the fulfilment of these objectives as the project progresses and thus demonstrate the feasibility and impact of the project results.

Fig. 2 shows the focus of every individual use case. They vary in: main focus (e.g., from transparency to quality improvement), setting (from a single product to a product line to multiple independent software products), domain (from highly privacy-aware systems to telecommunication networks) and process framework (from Scrum to ad hoc methods). Their diversity is both an opportunity and a challenge for the solutions provided in the project.

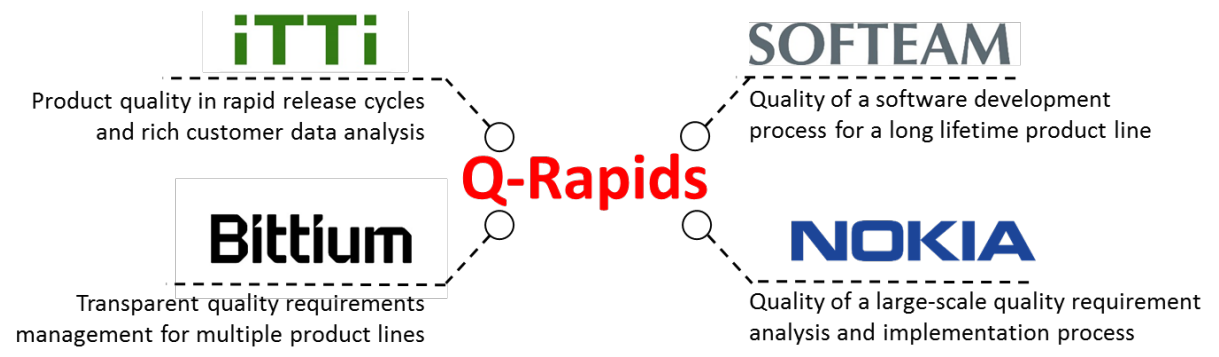

Fig. 2. Q-Rapids use cases

\section{Current State}

The Q-Rapids project was organized into five phases:

- Phase 1: project set-up (months 1-6). Main result: the Q-Rapids platform reference architecture, together with an implementation plan and the use cases specification.

- Phase 2: proof of concept (months 7-15). Main result: a first integrated version of the Q-Rapids platform, with simple techniques available. 
- Phase 3: consolidated framework (months 16-24). Main result: Q-Rapids platform integrating more powerful techniques and methods, with increasing validation from the use cases.

- Phase 4: final framework (months 25-33). Main result: final solution offering full functionality and fully-fledged associated processes.

- Phase 5: project finalization (months 34-36). Main result: packaging of the final solution.

At the moment of writing this report, Q-Rapids is entering in the final phase. The current framework is completed and the summative evaluation is being reported. A detailed overview of the project is described in [2].

\section{$5 \quad$ Achieved Outcomes}

The main outcomes of the Q-Rapids project are the Q-Rapids platform and Q-Rapids process.

The Q-Rapids platform is an advanced data-driven platform to manage quality in Agile and Rapid Software Development. Details on the consolidated version (phase 3) are described in [2]. Q-Rapids platform assists decision makers to make informed decisions by means of:

- Assessing the quality of the software under development at different abstraction levels. The platform relies on a quality model $(\mathrm{QM})$ to define three abstraction levels: key strategic indicators, project and process factors, and metrics [3]. The key strategic indicators can be assessed qualitatively defining a Bayesian Network based on the factors impacting on them [4].

- Predicting future quality levels through multiple predictive methods [5].

- Semiautomatically generating Quality Requirements to improve the quality of the software if such quality goes below some specific thresholds [6].

- Providing what-if analysis simulating the quality levels of the software given a particular scenario (e.g. the addition of a new quality requirement).

The Q-Rapids process is a layered process that relies on the Q-Rapids platform to support the quality management during the development process. The process includes how to use the Q-Rapids platform at the different development activities at three levels: product, release, and sprint. As part of the process development, we defined some process metrics to support the assessment of the development process performance [7].

Q-Rapids platform addresses objective $G 01$ and Q-Rapids process and process metrics address $G 02$. All assets together address objective G03.

\section{Why participating in PROFES 2019 European Project Space?}

As mentioned above, Q-Rapids is arriving to its end and in particular, by the time of the PROFES 2019 conference, it will be definitively over. Therefore, the main interest of the consortium is to demonstrate the final platform hoping that some other project, in earlier development stages, may be interested in adopting it. The code is open source and available in GitHub (https://github.com/q-rapids) with permissive licenses, and 
some of the partners are willing to collaborate in future endeavours. In fact, conversations with the ITEA-3 VISDOM project (https://itea3.org/project/visdom.html) are in place to use Q-Rapids in the context of DevOps processes analysis.

\section{Conclusions}

In this work, we have presented the objectives and current state of the Q-Rapids H2020 project. More information is available in the project website, www.q-rapids.edu. Components are available at https://github.com/q-rapids.

\section{Acknowledgments}

This work is a result of the Q-Rapids project, which has received funding from the European Union's Horizon 2020 research and innovation programme under grant agreement No 732253.

\section{References}

1. Liliana Guzmán, Marc Oriol, Pilar Rodríguez, Xavier Franch, Andreas Jedlitschka, Markku Oivo: How Can Quality Awareness Support Rapid Software Development? - A Research Preview. REFSQ 2017: 167-173.

2. Xavier Franch, Lidia Lopez, Silverio Martínez-Fernández, Marc Oriol, Pilar Rodríguez and Adam Trendowicz: Quality-aware Rapid Software Development: The Q-Rapids Project. TOOLS50+1.

3. Lidia López, Silverio Martínez-Fernández, Cristina Gómez, Michal Choras, Rafal Kozik, Liliana Guzmán, Anna Maria Vollmer, Xavier Franch, Andreas Jedlitschka: Q-Rapids Tool Prototype: Supporting Decision-Makers in Managing Quality in Rapid Software Development. CAiSE Forum 2018: 200-208.

4. Silverio Martínez-Fernández, Anna Maria Vollmer, Andreas Jedlitschka, Xavier Franch, Lidia López, Prabhat Ram, Pilar Rodríguez Marín, Sanja Aaramaa, Alessandra Bagnato, Michal Choras, Jari Partanen: Continuously Assessing and Improving Software Quality With Software Analytics Tools: A Case Study. IEEE Access 7: 68219-68239 (2019)

5. Martí Manzano, Emilia Mendes, Cristina Gómez, Claudia Ayala, Xavier Franch: Using Bayesian Networks to estimate Strategic Indicators in the context of Rapid Software Development. PROMISE 2018 : 52-55

6. Martí Manzano, Claudia Ayala, Cristina Gomez, Lidia López: A software service supporting software quality forecasting. DSQA 2019.

7. Marc Oriol, Pertti Seppänen, Woushet Behutiye, Carles Farré, Rafal Kozik, Silverio Martínez, Pilar Rodríguez, Xavier Franch, Sanja Aaramaa, Antonin Abhervé, Michal Choras, Jari Partanen. Data-driven elicitation of quality requirements in agile companies. QUATIC 2019.

8. Prabhat Ram, Pilar Rodríguez and Markku Oivo. Software Process Measurement and Related Challenges in Agile Software Development: A Multiple Case Study. PROFES 2018: 272-287. 